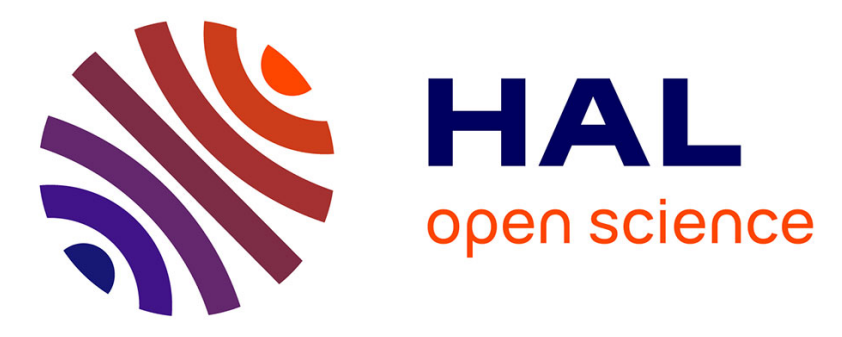

\title{
Hybrid Wireless Sensor Networks: A Prototype
} Alá Khalifeh, Novella Bartolini, Simone Silvestri, Giancarlo Bongiovanni, Anwar Al-Assaf, Radi Alwardat, Samer Alhaj-Ali

\section{To cite this version:}

Alá Khalifeh, Novella Bartolini, Simone Silvestri, Giancarlo Bongiovanni, Anwar Al-Assaf, et al.. Hybrid Wireless Sensor Networks: A Prototype. 17th IFIP Conference on Human-Computer Interaction (INTERACT), Sep 2019, Paphos, Cyprus. pp.549-553, 10.1007/978-3-030-29390-1_35 . hal-02877643

\section{HAL Id: hal-02877643 \\ https://hal.inria.fr/hal-02877643}

Submitted on 22 Jun 2020

HAL is a multi-disciplinary open access archive for the deposit and dissemination of scientific research documents, whether they are published or not. The documents may come from teaching and research institutions in France or abroad, or from public or private research centers.
L'archive ouverte pluridisciplinaire HAL, est destinée au dépôt et à la diffusion de documents scientifiques de niveau recherche, publiés ou non, émanant des établissements d'enseignement et de recherche français ou étrangers, des laboratoires publics ou privés.

\section{(c)(1)}

Distributed under a Creative Commons Attribution| 4.0 International License 


\title{
Hybrid Wireless Sensor Networks: a Prototype
}

\author{
Ala' Khalifeh ${ }^{1}$, Novella Bartolini ${ }^{2}$, Simone Silvestri ${ }^{3}$, Giancarlo Bongiovanni ${ }^{2}$, \\ Anwar Al-Assaf ${ }^{4}$, Radi Alwardat ${ }^{5}$, and Samer Alhaj-Ali ${ }^{5}$ \\ 1 German Jordanina University, Jordan \\ ala.khalifeh@gju.edu.jo \\ 2 Sapienza University of Rome, Italy \\ \{novella, bongiovanni\}@di.uniroma1.it \\ 3 University of Kentucky \\ silvestri@cs.uky.edu \\ 4 Arab Robotics and AI Organization, Jordan \\ Muaddi130yahoo.com \\ 5 The King Abdullah II Design and Development Bureau (KADDB), Jordan \\ \{ralwardat, shajali\}@kaddb.mil.jo
}

\begin{abstract}
Wireless sensor networks (WSNs) are widely used to assist monitoring of an area of interest whenever manual monitoring by skilled personnel is not convenient if not prohibitive. In this paper, we propose a step ahead with respect to the current status of art, with the design and implementation of a novel networked architecture which integrates a set of static ground nodes, an Unmanned Ground Vehicle (UGV), and an Unmanned Aerial Vehicle (UAV) in a unique monitoring system. All these devices are equipped with sensors, a microcontroller and a wireless communication module such that they are capable of covering an AoI in the terrestrial and aerial dimension. In the proposed architecture, the mobile robot can inspect areas which have no fixed ground sensors, whereas sensors installed on the UAV cover the ground areas from above, ensuring wider coverage. Once data is collected by the ground sensors, it is sent via the UAV to a remote processing center, which elaborates the gathered data for decision making.
\end{abstract}

Keywords: Wireless Sensor Networks (WSN) · Unmanned Ground Vehicle (UGV) • Unmanned Aerial Vehicle (UAV) • Remote Monitoring • Decision Making .

\section{Introduction}

Wireless sensor networks consist of a group of nodes deployed over an area of interest to monitor a variety of environmental features, depending on the application. As coverage and connectivity remain an issue for static sensor network deployments $[1,2]$, and severely limit their applicability to small and safe areas of interest, we propose a novel monitoring architecture which encompasses the

\footnotetext{
* This work is supported by NATO - North Atlantic Treaty Organization, under the
} SPS grant G4936 SONiCS: Hybrid Sensor Network for Emergency Critical Scenarios 
above limitations. Our monitoring system integrates static and mobile devices cooperating with each other to ensure a flexible and quickly deployable monitoring network, to be used in a wide range of operating settings, including health crop monitoring for agriculture as well as monitoring of critical scenarios for homeland security. For example, the proposed system can be used to monitor wildfires, or areas which have been exposed to chemical plumes, gas leakage, radiation or to an intentional attack with nuclear or chemical weapons, in case of war, which is dangerous to explore by humans, thus the proposed system provides safe, efficient and convenient way for remote sensing, that can be used by decision makers for assessing the safety of the monitored area of interests. In the literature, several network architectures have been proposed to achieve this purpose. The authors in [3] proposed to utilize UAVs in conjunction with static sensors on the ground to improve the quality of packet routing. Another work that utilizes UAVs to assist ground sensors is presented in [4], where UAVs are used to spray crops while adaptively planning their route based on the findings of the ground sensors. Some researchers modeled WSN networks that use both ground and aerial nodes as a three-dimensional network, focusing on the study of connectivity and coverage problems [5]. Unlike previous approaches, our network architecture utilizes both static and mobile terrestrial sensors, as well as aerial sensing vehicles, such that the three elements work collaboratively to provide comprehensive sensing and coverage of the area of interest, and efficient communications with the processing center. Differently from previous studies [6-8] mostly based on simulations, this work focuses on the actual implementation of such a network: in Section II we describe our monitoring architecture, while in Section III we conclude the discussion and outline future research directions.

\section{WSN Architecture and Implementation}

As shown in Fig. 1, the proposed monitoring network consists of three types of nodes: static nodes, an Unmanned Ground Vehicle (UGV) and an Unmanned Aerial Vehicle (UAV). The monitoring process consists of two main stages. In stage 1 , sensed data are captured and stored by the static nodes, sent to the UGV node, which acts as a sink node, then transferred to the UAV node. After that, the data is transferred by the UAV to the remote data analysis and decision making center. Where responsible people who are observing the AoI will analyze the data and use it for decision making process.(e.g. declaring the AoI to be safe for humans or not.)

\subsection{Static nodes}

Figure 1 (a) shows static nodes to be deployed on the ground along the area of interest. These nodes can communicate either directly with each other or through the mobile nodes (the UGV and the UAV). The static node consists of a microcontroller, sensor interface board, sensors and battery as shown in Fig.2. 


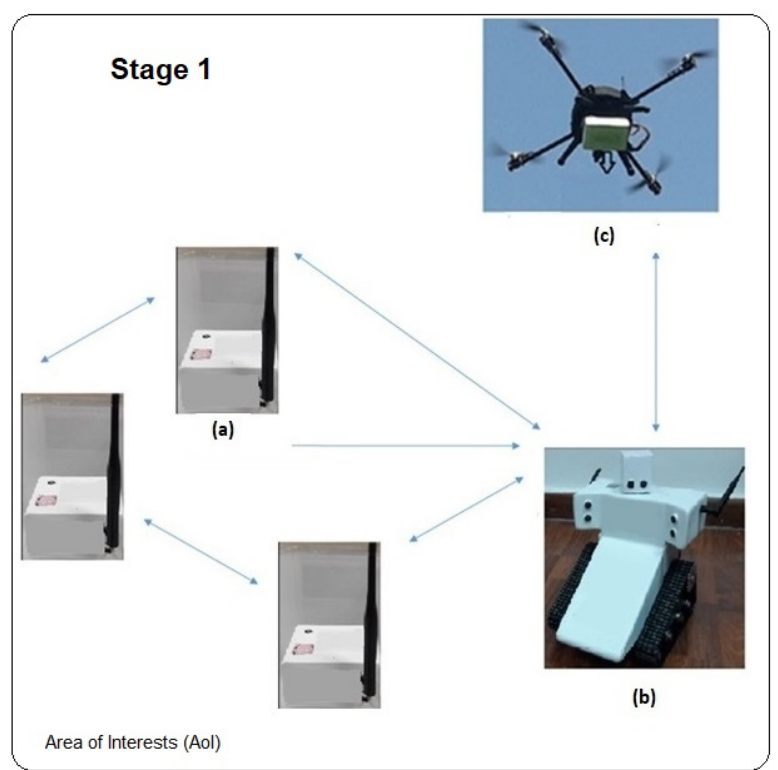

Stage 2

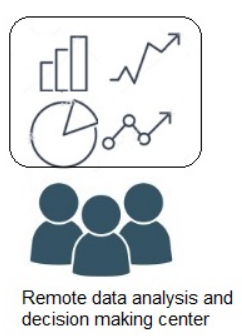

Fig. 1: Monitoring architecture: static sensor nodes (a), a UGV (b), and a UAV (c).

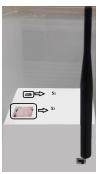

(a)

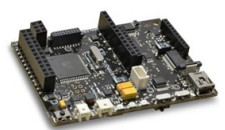

(b)

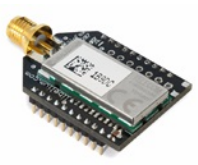

(c)

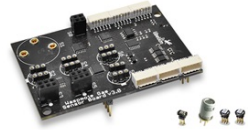

(d)

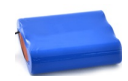

(e)

Fig. 2: A static node (a) and its components: a microcontroller (b), a wireless transceiver (c), sensors and related interface board (d), and battery (e).

\subsection{Unmanned Ground Vehicle (UGV)}

The coverage and communication capabilities of our network are enhanced by using a mobile terrestrial device, namely the UGV shown in Fig. 3. The UGV is provided with ultrasonic sensors (marked in blue) to detect and avoid obstacles. It also has three antennas A1, A2, and A3, used to communicate with the UAV, static nodes, and the Global Position System (GPS), respectively. This robot is 
capable of autonomous path planning, namely it can explore the area and avoid obstacles and objects found along its path using ultrasonic sensors.

The functionalities of the UGV can be summarized in three main tasks. The first one is to communicate with the fixed ground sensors for collecting data from them thus acting as a cluster head. The second task is to conduct a survey of the area, especially for areas where there are no static sensors. The third task of these mobile robots is to send the data collected from the ground sensors to the $\mathrm{UAV}$, which is then transferred via the UAV to the remote processing center to make the appropriate decisions.

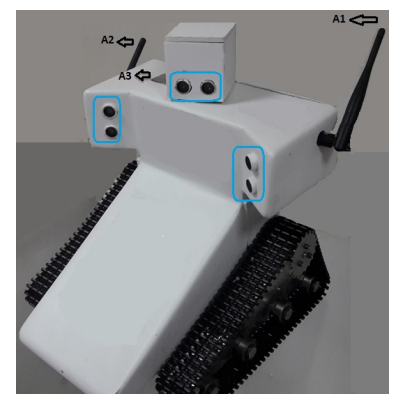

Fig. 3: Unmanned Ground Vehicle (UGV).

\subsection{Unmanned Aerial Vehicle}

The third fundamental component of our monitoring system is the Unmanned Aerial Vehicle shown in Fig.4. The ground sensing system is complemented with an aerial sensing node onboard of a UAV which monitors the area from above, capturing features that could not be detected by ground devices only. In addition, this UAV is equipped with a wireless module that enables communications with mobile ground sensors, thus receiving the data gathered from the ground sensors and transferring it to the processing center for data analysis and decision making.

\section{Conclusion}

In this paper, we presented the implementation of a heterogeneous wireless sensor network that consists of static, mobile and flying nodes, that can work collaboratively to cover an area of interest, and provide the sensed data for a remote center for data analysis and decision making, which not only saves people from investigating the area of interest personally thus saving time and effort, but also provides a convenient and safe monitoring process. As a future work, we are 


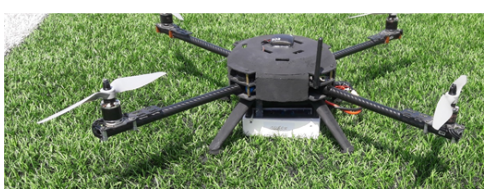

(a)

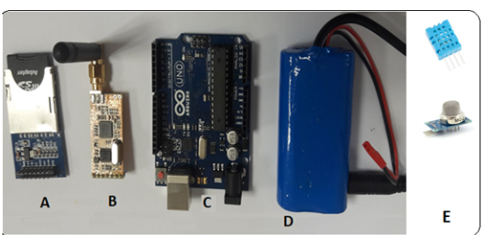

(b)

Fig. 4: A UAV with a sensor node on its base (a). Components of the UAV node (b) consisting of: an SD card for storing the data (A), a wireless transceiver (B), a microcontroller $(\mathrm{C})$, a battery (D), and sensors (E).

working on building an intelligent Human Computer Interaction (HCI) System that utilizes the developed data sensing and collection systems, which can make decisions and recommendations based on both the human and computer decision. Further, the energy consumption of the UGV and sensor nodes needs more optimization.

\section{References}

1. N. Bartolini, T. Calamoneri, T. La Porta, C. Petrioli, and S. Silvestri, "Sensor activation and radius adaptation (SARA) in heterogeneous sensor networks," $A C M$ Trans. Sen. Netw., vol. 8, no. 3, pp. 24:1-24:34, Aug. 2012.

2. N. Bartolini, T. Calamoneri, A. Massini, and S. Silvestri, "On adaptive density deployment to mitigate the sink-hole problem in mobile sensor networks," Mob. Netw. Appl., vol. 16, no. 1, pp. 134-145, Feb. 2011.

3. D. L. Gu, G. Pei, H. Ly, M. Gerla, B. Zhang, and X. Hong, "Uav aided intelligent routing for ad-hoc wireless network in single-area theater," in Wireless Communications and Networking Confernce, 2000. WCNC. 2000 IEEE, vol. 3. IEEE, 2000, pp. $1220-1225$.

4. F. G. Costa, J. Ueyama, T. Braun, G. Pessin, F. S. Osório, and P. A. Vargas, "The use of unmanned aerial vehicles and wireless sensor network in agricultural applications," in Geoscience and Remote Sensing Symposium (IGARSS), 2012 IEEE International. IEEE, 2012, pp. 5045-5048.

5. A. M. Al tahan and M. K. Watfa, "A position-based routing algorithm in 3d sensor networks," Wireless Communications and Mobile Computing, vol. 12, no. 1, pp. $33-52,2012$.

6. A. F. Khalifeh, M. AlQudah, R. Tanash, and K. A. Darabkh, "A simulation study for uav- aided wireless sensor network utilizing zigbee protocol," in 2018 14th International Conference on Wireless and Mobile Computing, Networking and Communications (WiMob), Oct 2018, pp. 181-184.

7. A. F. Khalifeh, H. Salah, S. Alouneh, A. Al-Assaf, and K. A. Darabkh, "Performance evaluation of digimesh and zigbee wireless mesh networks," in 2018 International Conference on Wireless Communications, Signal Processing and Networking (WiSPNET). IEEE, 2018, pp. 1-6.

8. K. A. Darabkh, S. M. Odetallah, Z. Al-qudah, A. Khalifeh, and M. M. Shurman, "Energy-aware and density-based clustering and relaying protocol (ea-db-crp) for gathering data in wireless sensor networks," Applied Soft Computing, 2019. 